\title{
Extend the Zone and Beat the Resistance
}

\section{Julio C Hernández*}

Hernández Molinar Consulting, Caracas, Venezuela

\section{Introduction}

According to Klein [1] and Fairbairn [2] and others, the early The Comfort Zone is a term that is currently very fashionable, it speaks of how negative it is for the individual to remain always within the comfort zone, since this generates comfort, and makes the individual always have the same behaviors and to obtain the same results, it is also suggested that the comfort zone should come out or change, but in my view, an abrupt change or "exit" of the zone would generate a great resistance to change and would force the individual to return to the area or the situation in which it was already, since accompanied by resistance to change goes a great belief to "I cannot do that, I will go wrong, etc." that limits the acceptance of that change.

This has encourage to write these lines since in many cases our area can cause negative effects to our life, just as when the individual wants to change, a resistance arises, since this change can affect behaviors or behaviors to perform daily and I do not want to change immediately, hence the proposal stated as: "Expanding the comfort zone decreases resistance to change".

\section{Development}

The Comfort Zone is defined as a behavioral state in which the individual operates in a condition of "neutral anxiety", using a series of behavior to achieve a constant level of meaningless risk yields [1]. It is the set of all those physical or psychological places in which our mind feels safe, comfortable and protected [2].

According to these ideas and others I have collected in recent months we dare to define the Comfort Zone as a state where we feel comfortable, relaxed, safe, where we perform the same behavior and get the same results without taking any type of risk, is the place where we already know that everything is going to happen according to what we want.

Based on these definitions we came up with the following question: Is the comfort zone a reality or a limiting belief?

To try to answer this question, let us initially define what is Belief? Belief, to believe, is the firm settlement and conformity with something [3]. The individual thinks that only in what he believes is what exists, limiting the possibility of assessing if there is something more or something different, based on a belief the individual believes himself unable to do something (perform some activity) or develop a new capacity or strengthen an existing one.

Associated with the change of beliefs or as part of the "Expansion of the Zone" always appear mental saboteurs or self-sabotage, these work in favor of the current area in order to block any kind of change. Similarly if you do not correctly define the change you want to give this can lead to frustration or depression, for example, a woman wants to lose weight because she thinks that would be much more attractive to men and you would have many options to achieve your dream that is marry the perfect man, when this is not fulfilled the woman will feel that the change she gave to her life was useless.

Beliefs have a very close relationship with behavior, if a person believes strongly in something; behave in a manner consistent with their belief. For Hoffmann W Belief is A set of ideas - which we consider to be true and not necessarily have a rational origin - that express a negative or positive vision about the global or partial, external and / or subjective reality of being [4]. Beliefs can become so strong that you are limited in some activity that you want or know that you must change; beliefs have a direct relationship with the behavior of individuals. You can teach spelling to anyone as long as you are alive and able to return information. However, if the person truly believes that he cannot do something, he will find some unconscious way of preventing change from occurring [5].

It is important to identify if the preexisting belief is limiting and the best is that it can be changed progressively or if this belief could be enhanced by improving the capabilities of the individual. A very clear example of what was mentioned by Abraham Maslow where a psychiatrist treated a man who believed to be a corpse. Despite all the logical arguments of the psychiatrist, man persists in his belief. Finally, in a flash of inspiration, the psychiatrist asked him, "Can corpses bleed?" The patient replied, "That's absurd! The corpses do not bleed. "After asking for permission, the psychiatrist punctured his finger with a needle and extracted a drop of bright red blood. The patient stared at his finger, completely astonished, and at once exclaimed, "Let me be ashamed! Now it turns out that the corpses bleed! [5].

\section{Conclusion}

As positive changes are incorporated in order to achieve a short- or medium-term life goal, we are "Expanding the Comfort Zone", these changes must be gradual and at the same time motivate these changes by applying rewards to increase the satisfaction and motivation to achievement. For example, participation in public sports competitions or activities in order to evaluate improvements, purchase of articles or tools that helps to improve performance, among others.

It is important at the moment when it is decided to "Expand Comfort Zone" to define the current or present situation and to visualize the desired or future situation, this will help to identify the capacities to strengthen or improve the resources to be needed with in order to achieve the stated objective.

\section{References}

1. White A (2008) From Comfort Zone to Performance Management

2. Celis S (2017) Personal Development.

3. Royale AE (2010) Dictionary. Madrid.

4. Hoffmann W (2013) Limiting Beliefs. Melvin CA, Caracas.

5. Robert D (2008) Identification and Change of Beliefs.

*Corresponding author: Julio C Hernández, Practitioner PNL, Hernández Molinar Consulting, Caracas, Venezuela, Tel: + 58-412-2839062; E-mail: coachjuliohernandez@gmail.com

Received: October 23, 2017; Accepted: November 04, 2017; Published: November 11, 2017

Citation: Hernández JC (2017) Extend the Zone and Beat the Resistance. Clin Exp Psychol 3: 173. doi: 10.4172/2471-2701.1000173

Copyright: ( 2017 Hernández JC. This is an open-access article distributed under the terms of the Creative Commons Attribution License, which permits unrestricted use, distribution, and reproduction in any medium, provided the original author and source are credited. 